\title{
SPRAY IN AUTOMOTIVE APPLICATIONS: PART II
}

\author{
Guest Editors
}

Sibendu Som

\section{PREFACE}

The next-generation low-emission flexible fuel automobile engine will be developed by closely coordinating high-fidelity experiments and predictive simulation capabilities. Fuel injection and subsequent spray development will still play a key role in the optimization of engine performance and efficiency since they directly influence fuel-air mixing, ignition and combustion characteristics, and pollutant formation. These, in turn, will influence the final cost to the customer since they dictate the need for additional engine technologies such as after-treatment, engine boosting systems, and more.

Fuel spray has a profound influence on current and future automobile engines and this issue of Atomization and Spray is focused on improving our understanding of the processes governing spray formation under internal combustion engine conditions. There is a need for developing predictive spray models at a reasonable cost which will facilitate the co-optimization of fuel injectors and engine geometries. Towards this end, in general, there is definitely room for using high-fidelity experimental data generated by the wide variety of conditions encountered in spark and compression ignition engines.

Researchers in all disciplines at the forefront of fuel spray research have been invited to contribute to this issue. The papers provide the reader with a broad overview of not only current work, but also future work required to meet remaining challenges. The contents of part 2 make up issues 5 and 6 and focus on experimental work on sprays for automobile applications.

Issues 5 and 6 are made up of the following six papers:

1. The paper by Hulkkonen et al. (2014) focuses on experimental studies of conical and cylindrical diesel injector nozzle orifices. For more than a decade now, researchers have tried to control in-nozzle cavitation and turbulence by modifying the orifice geometry to optimize the ensuing spray. The main objective of the study was to examine the influence of conicity on spray development when the orifice outlet diameter was kept constant. The authors measured spray penetration, cone 
angle, and mass flow rate at the orifice exit under non-evaporating conditions at ambient densities relevant to diesel engine operation. The findings provide insight and some are in disagreement with previously published literature.

2. The article by Moon et al. (2014) studies the morphology of the emerging sprays from 6-hole and 2-hole diesel injectors. These injectors are characterized by different needle control mechanisms which resulted in different jet flows and breakup processes at different needle lifts. The short $\mathrm{x}$-ray pulses from a third-generation synchrotron source possessing the potentials to freeze the motion of the highspeed sprays and to detect the liquid/gas interfaces inside the sprays were employed to extract the morphological information and dynamics of the sprays in the near-field using the single- and multi-exposed $\mathrm{x}$-ray phase-contrast images. A highly expanding cone-shaped spray was observed at the low needle lifts, but the degree of flow expansion was diminished at higher needle lifts due to attenuated vortex strength at the these lifts. This study clearly highlights the need to understand the needle-dynamics for better understanding the ensuing fuel spray.

3. The contribution by Battistoni et al. (2014) is a collaborative work between experimentalist and modelers to better understand the effect of non-condensable gases on void fraction inside a cavitating nozzle. Since quantitative measurements of void fractions are limited in literature, this unique experimental data from Argonne's Advanced Photon Source provides a comprehensive data-set for validating nozzle flow models. While the technique can measure void fractions inside a polycarbonate injector, the distinction between voids due to cavitation or non-condensable gas expansion is not clear. The authors first provide a rigorous validation of the Homogeneous Relaxation Model and then demonstrate that simulations can distinctly capture regions of non-condensable gas expansion and cavitation due to phase change. This study highlights the need to know and control the amount of non-condensable gases in fuel.

4. The article by Pickett et al. (2014) is an uncertainty quantification study of the liquid penetration of evaporating sprays under diesel-like conditions. Ten different light-scatter and extinction diagnostics were used to measure the liquid length. Measurements showed that light scattering is sensitive to the orientation of the illumination source. Nevertheless, the scattered intensity for these set-ups can be normalized to provide better estimation of the liquid length. Beam steering due to differences in refractive index gradients influence light-extinction diagnostics. The authors accommodate for beam steering effects using a small laser beam with large collection optics. The possibility of the existence of supercritical mixtures is acknowledged; however, Mie-scatter theory is applied for droplet diameters between 0.1 and $10 \mu \mathrm{m}$. With a measured liquid path length of $1.4 \mathrm{~mm}$, the upper boundary of the path-length-averaged liquid volume fraction was estimated to be 
about $0.15 \%$. Such uncertainty quantification studies provide CFD modelers with more quantitative estimates for developing and validating spray models.

5. The paper by Magnotti and Genzale (2014) presents a new spray model validation methodology using liquid-phase extinction measurements. Lagrangian spray models are usually validated against spray images based on elastic or Mie scattering of light from liquid structures and droplet clouds. These techniques do not typically detect absolute liquid boundaries. However, simulations the absolute liquid boundaries can be detected. Hence it is preferable to use these liquid volume fraction thresholds to similarly define the liquid boundary from Mie-scatter images. This study is a key step towards better understanding of experimental findings when defining the spray boundary accurately from optical measurements. It is expected that the spray modeling community will adopt more consistent strategies for comparing simulation results with experiments such as the one presented here.

6. The contribution by Pastor et al. (2014) is focused on the development of a onedimensional model to predict the liquid length and spray penetration for blends of single-component diesel fuel surrogates. This study is an advancement of the author's previous work on developing one-dimensional spray models for single component diesel fuel surrogates. The model development necessitated the implementation of a high-pressure liquid-vapor equilibrium model together with the hypothesis of a real-gas mixture to calculate the partial enthalpy of each component in the blend. The one-dimensional model is extensively validated against data for liquid length and vapor penetration for blends of n-decane and n-hexadecane under different conditions. The authors further tested the model against a blend of six single-component surrogates. Such one-dimensional models are often used by experimentalists and modelers to gain a basic understanding of expected spray behavior, and thus, is expected to be used extensively in the research community. 